\title{
A Patient with Sjogren's Syndrome and Subsequent Diagnosis of Inclusion Body Myositis and Light-Chain Amyloidosis
}

\author{
Jason Hom, MD ${ }^{1,2}$, Shruti Marwaha, $P h D^{1,3}$, Anna Postolova, MD4, Jessie Kittle, $M D^{2}$, \\ Rosaline Vasquez, MD' ${ }^{5}$, Jean Davidson, $P h D^{7}$, Jennefer Kohler, MS, $L C G C^{7}$, Annika Dries, BS , \\ Liliana Fernandez-Betancourt, $M D^{7}$, Marta Majcherska, $B S^{7}$, Joanna Dearlove, $M D^{6}$, \\ Shyam Raghavan, $\mathrm{MD}^{7}$, Hannes Vogel, MD, $P h D^{7}$, Jonathan A. Bernstein, $M D, P h D^{1,8}$, \\ Paul Fisher, $M D^{1,9}$, Euan Ashley, MRCP, $P h D^{1,3}$, Jacinda Sampson, MD, $P h D^{1,6}$, and \\ Matthew Wheeler, MD, PhD ${ }^{1,3}$ Undiagnosed Diseases Network
}

\begin{abstract}
'Stanford Center for Undiagnosed Diseases, Stanford University School of Medicine, Stanford, CA, USA; 2 Division of Hospital Medicine, Department of Medicine, Stanford University School of Medicine, Stanford, CA, USA; ${ }^{3}$ Division of Cardiovascular Medicine, Department of Medicine, Stanford University School of Medicine, Stanford, CA, USA; ${ }^{2}$ Division of Immunology and Rheumatology, Stanford University School of Medicine, Stanford, CA, USA; ${ }^{5}$ Division of Primary Care and Population Health, Department of Medicine, Stanford University School of Medicine, Stanford, CA, USA; ${ }^{6}$ Department of Neurology, Stanford University School of Medicine, Stanford, CA, USA; ${ }^{7}$ Department of Pathology, Stanford University School of Medicine, Stanford, CA, USA; ' ${ }^{8}$ Division of Medical Genetics, Department of Pediatrics, Stanford University School of Medicine, Stanford, CA, USA; ${ }^{9}$ Division of Child Neurology, Department of Neurology, Stanford University School of Medicine, Stanford, CA, USA.
\end{abstract}

We discuss a challenging case of a 58-year-old VietnameseAmerican woman who presented to her new primary care provider with an 8-year history of slowly progressive dysphagia, hoarseness, muscle weakness with associated frequent falls, and weight loss. She eventually reported dry eyes and dry mouth, and she was diagnosed with Sjogren's syndrome. Subsequently, she was additionally diagnosed with inclusion body myositis and gastric light-chain (AL) amyloidosis. Although inclusion body myositis has been previously associated with Sjogren's syndrome, inclusion body myositis is rare in non-Caucasians, and the trio of Sjogren's syndrome, inclusion body myositis, and AL amyloidosis has not been previously reported. Sjogren's syndrome is a systemic autoimmune condition characterized by ocular and oral dryness. It is one of the most common rheumatologic disorders in the USA and worldwide. Early diagnosis of Sjogren's is particularly important given the frequency and variety of associated autoimmune diseases and extraglandular manifestations. Furthermore, although inclusion body myositis has a low prevalence, it is the most common inflammatory myopathy in older adults and is unfortunately associated with long delays in diagnosis, so knowledge of this disorder is also crucial for practicing internists.

KEY WORDS: Sjogren's syndrome; inclusion body myositis; amyloidosis; immunophenotyping.

Prior Presentations We presented an earlier version of the manuscript as a poster at the American College of Physicians Northern California Meeting in 2016.

Electronic supplementary material The online version of this article (https://doi.org/10.1007/s11606-019-04931-w) contains supplementary material, which is available to authorized users.

Received October 23, 2017

Revised April 3, 2018

Accepted February 22, 2019

Published online March 18, 2019
J Gen Intern Med 34(6): 1058-62

DOI: $10.1007 / \mathrm{s} 11606-019-04931-\mathrm{w}$

(c) Society of General Internal Medicine 2019

\section{CASE PRESENTATION}

A 58-year-old Vietnamese-American woman with a remote history of treated scrofula established care with a new primary care provider at the resident clinic of a tertiary academic institution. She reported an 8-year history of slowly progressive dysphagia, hoarseness, proximal lower extremity muscle weakness (with associated frequent falls), and weight loss, with a body mass index (BMI) that had gradually decreased from 20 to 15 . Her recent work-up included an esophagogastroduodenoscopy (EGD) with biopsies positive for Helicobacter pylori and esophageal manometry consistent with diffuse esophageal spasm; treatment with triple therapy and a trial of diltiazem did not improve her dysphagia or weight loss. Her prior work-up also included cancer screening, with a recent negative colonoscopy, mammogram, and Pap smear. Her new primary care provider additionally sent serum and urine protein immunofixation electrophoresis studies (SPIE and UPIE, respectively), which were also negative.

Concerned by her dysphagia and weight loss, her new primary care provider referred her for a gastroenterology second opinion, with a repeat EGD and manometry demonstrating diffuse esophageal spasm with unremarkable biopsies. On multiple subsequent visits to primary care, her providers noted subtle bilateral proximal lower extremity weakness on exam, which was attributed to poor nutritional intake and overall deconditioning. The patient was referred to physical and occupational therapy.

At a subsequent visit, in addition to her aforementioned ongoing symptoms, she reported dry eyes and mouth. Her PCP (primary care provider) referred her to rheumatology and 
ophthalmology. A Schirmer test for dry eyes was positive. Serologies were significant for positive anti-nuclear antibody (ANA) titer of 1:2560 (normal < 1:40) and positive anti-SSA/Ro and anti$\mathrm{SSB} / \mathrm{La}$ at $>100 \mathrm{U} / \mathrm{ml}$ (normal $<20 \mathrm{U} / \mathrm{ml}$ ). She was diagnosed with Sjogren's syndrome, for which was started on hydroxychloroquine and low-dose prednisone (5 mg daily). Salivary gland biopsy was considered but deferred.

As her long-standing proximal muscle weakness continued to progress, she was eventually re-referred to neurology, and initial evaluation included an electromyography, which showed findings consistent with multifocal polyradiculopathy versus motor neuron disease. Creatine kinase (CK) was checked on multiple visits and always within normal limits $(97,142,125 \mathrm{U} / \mathrm{L})$. Lactate dehydrogenase (LDH) was $255 \mathrm{U} /$ $\mathrm{L}$, C-reactive protein (CRP) was $0.7 \mathrm{mg} / \mathrm{dL}$, and erythrocyte sedimentation rate (ESR) was $111 \mathrm{~mm} / \mathrm{h}$.

Eventually, the patient and her providers felt that her symptoms had started to progress more quickly, and her BMI reached a nadir of 14. Her PCP referred her to the Undiagnosed Diseases Network (UDN). ${ }^{1}$ The UDN is an initiative sponsored by the National Institutes of Health, with the goal of using coordinated multispecialist consultations and advanced testing (including researchbased genetic testing and immunophenotyping) to facilitate diagnosis of rare and novel diseases in clinically challenging patients, as well as to shed new insights into disease pathophysiology. Her case was reviewed and accepted at the UDN's Stanford Center for Undiagnosed Diseases, and she was subsequently hospitalized for expedited multi-specialty inpatient work-up.

During her hospitalization, a muscle biopsy of her right vastus lateralis was performed (Fig. 1). The biopsy revealed inflammatory myopathy with mitochondrial pathology, a condition within the spectrum of inclusion body myositis (IBM). Notably, Congo red stains on her muscle biopsy were negative for amyloid.

Due to progression of her dysphagia and malnutrition, a third EGD with biopsies was performed. Gastric biopsy revealed amyloid on Congo red staining (Fig. 2), with liquid chromatography tandem mass spectrometry showing a peptide profile consistent with light-chain (AL) amyloid deposition (lambda-type). Free light-chain kappa/lambda ratio was slightly elevated at 1.7 (with 1.6 as the upper limit of normal on our assay). The patient was referred to an amyloid specialist in the hematology department. Bone marrow biopsy was performed, without evidence of a plasma cell neoplasm or amyloid; however, her hematologists noted that this was difficult to interpret as the patient was on highdose steroids for her myositis at the time of the biopsy. Her prior EGDs 5 years prior had unrevealing biopsies, and multiple prior free light-chain kappa/lambda ratios had been normal $(1.1,1.5)$ 2 years prior, suggesting that the amyloid was a relatively new process or that her prior light-chain ratios had been confounded by low-dose prednisone for her Sjogren's. She was advised to start bortezomib (a proteasome inhibitor) and her steroid and methotrexate doses were titrated as well. Follow-up screening serum protein immunoelectrophoresis (SPIE), urine protein immunoelectrophoresis (UPIE), and free light-chain kappa/lambda ratio were all normal. Post-treatment CK and LDH were $475 \mathrm{U} / \mathrm{L}$ and $275 \mathrm{U} / \mathrm{L}$, respectively. Initially, she reported improvement in her strength, reporting to her neurologist that she felt that "her arm muscles [were] getting bigger," although her dysphagia persisted. She has continued on bortezomib but stopped plaquenil 23 months after starting it due to lack of benefit for her fatigue and weakness.

\section{DISCUSSION}

Sjogren's syndrome has been previously associated with IBM (Sjogren's syndrome-IBM), although this is relatively uncommon. ${ }^{2,}{ }^{3}$ Moreover, there are rare case reports of Sjogren's syndrome associated with $\mathrm{AL}$ amyloidosis, ${ }^{4}$ and monoclonal gammopathies are commonly associated with IBM. ${ }^{5}$ However, IBM is relatively rare in non-Caucasians, ${ }^{6}$ and neither the simultaneous presentation of Sjogren's, inclusion body myositis, and AL amyloidosis nor a unifying underlying pathogenesis for this trio of diseases has been described previously, to the best of our knowledge.

Numerous investigators attempted to explore shared pathophysiology between IBM and Sjogren's. From a genetic perspective, these two diseases have similar underlying predispositions based on HLA subtypes common to IBM, Sjogren's, and other autoimmune conditions. ${ }^{3,7,8}$ Indeed, muscle biopsies from IBM and salivary gland biopsies from Sjogren's reveal a mixture of $\mathrm{B}$ and $\mathrm{T}$ cells in both cases, with cytotoxic T cells present. ${ }^{9}$

In examining muscle biopsies from IBM, other investigators have examined whether amyloid beta deposition plays a role, hypothesizing that impaired proteasomes and autophagy lead to abnormal protein accumulation, inflammation, and myopathy. ${ }^{10}$ Of note, despite this hypothesis, amyloid has been inconsistently seen in muscle biopsies of IBM patients, ${ }^{11},{ }^{12}$ which some authors postulate is due to limited sensitivity from relatively small samples. ${ }^{12}$ For our patient, Congo red staining on skeletal muscle biopsy was negative. The hypothesized role of amyloid beta in IBM has not been linked to amyloid light-chain amyloidosis, as was seen in our patient's gastric biopsy. These prior studies have elucidated potential connections between (1) Sjogren's and IBM and between (2) IBM and amyloid, but uncertainty exists as to whether Sjogren's, AL amyloid, and IBM share a common link.

From a clinical perspective, IBM is the most common idiopathic inflammatory myopathy in adults over $50,{ }^{13-15}$ with a prevalence of 3.5/100,000 in this age group. ${ }^{6}$ Presenting symptoms include dysphagia and weakness, particularly in the wrist, finger flexors, and quadriceps. Pulmonary involvement is uncommon. ${ }^{16} \mathrm{CK}$ levels in inclusion body myositis can be normal (as was the case for our patient) or substantially elevated up to 20 times the normal limit. ${ }^{6}$ As in this case, muscle biopsy is crucial for the diagnosis.

Unfortunately, in the setting of an often insidious onset of symptoms and a broad differential for weakness, IBM is difficult to diagnose, with prior studies suggesting an average duration of 5-8 years from symptom onset to diagnosis. ${ }^{6}$ 


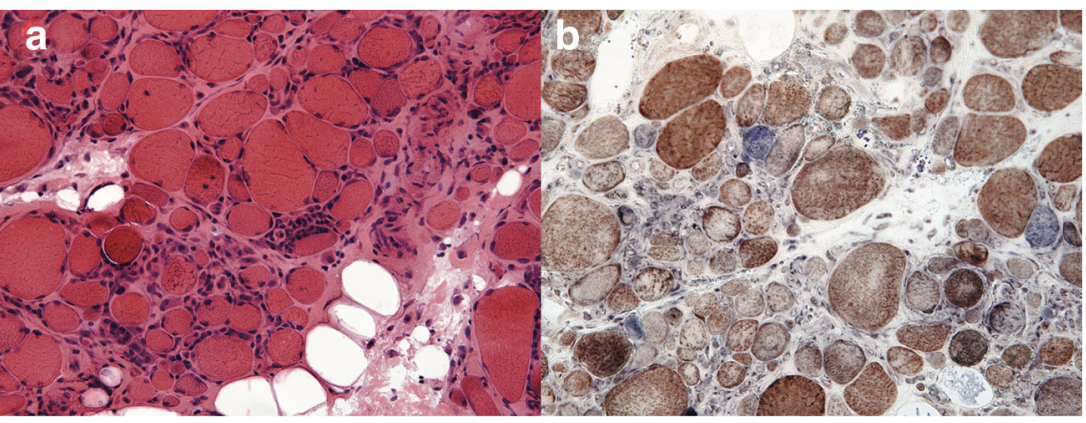

Figure 1 Muscle biopsy slides. Part (A) (on the left) reflects an H\&E stain, while part (B) (on the right) reflects a trichrome stain. The patient's muscle biopsy demonstrated endomysial inflammation without rimmed vacuoles and with cytochrome oxidase negative fibers, which provided support for the diagnosis of inflammatory myopathy with mitochondrial pathology, a condition within the spectrum of inclusion body myositis.

Given this data, our patient's diagnosis 5 years after initial presentation is not entirely unexpected. However, after she was diagnosed with Sjogren's (4 years after establishing care) and was noted to have concurrent slowly progressive weakness, inclusion body myositis could have been prioritized in the differential given its rare but known association with Sjogren's. ${ }^{9}$ To aid clinicians, there is emerging evidence that some clinical decision support systems such as Isabel (Isabel Healthcare Inc., Ann Arbor, MI, USA) or DxPlain (Massachusetts General Hospital Laboratory of Computer Science, Boston, MA, USA) can have significant diagnostic accuracy in complex cases such as the one presented. ${ }^{17,}{ }^{18}$ In general, clinical decision support systems will require substantial study, refinement, and validation prior to widespread clinical use; indeed, a recent systematic review and meta-analysis found substantial variability in the efficacy of clinical decision support systems, with such systems sometimes providing minimal benefits for complex cases. ${ }^{19}$

Unlike dermatomyositis or polymyositis, sporadic IBM is generally non-responsive to corticosteroids and is relatively difficult to treat. ${ }^{14}$ However, prior studies suggest that Sjogren's syndrome-IBM may be more responsive to immunosuppression than sporadic $\mathrm{IBM}^{20}$; in particular, IBM associated with connective tissue disorders may be responsive to methotrexate. ${ }^{9}$ Given these findings, our patient and her clinical providers were hopeful that she would derive benefit from methotrexate. Unfortunately, our patient has not experienced an overall clinical improvement with methotrexate for her Sjogren's syndrome-IBM and bortezomib for her AL amyloidosis, despite over 20 months of treatment with both agents. The difficulties with treating both sporadic IBM in general and with treating our particular patient highlight the need for
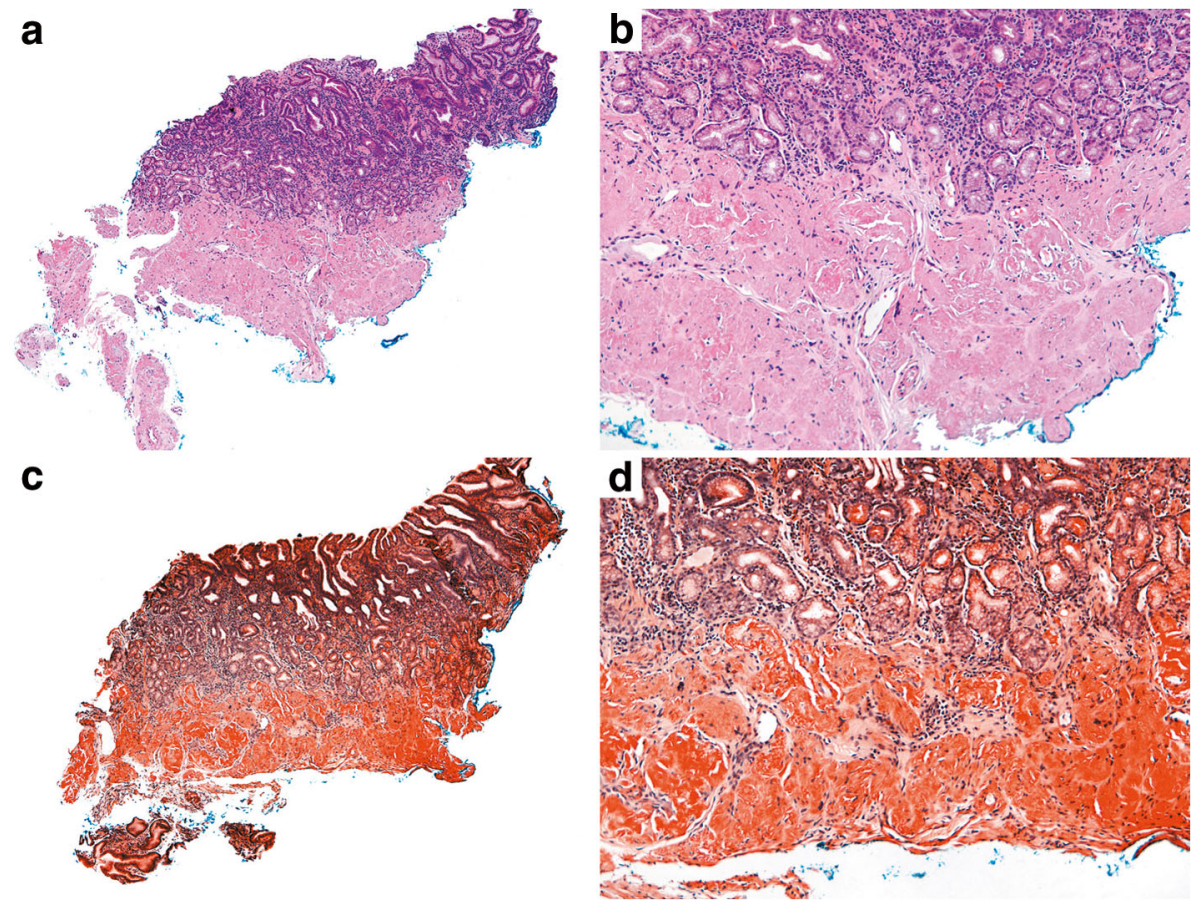

Figure 2 Gastric biopsy. Parts (A) and (B) reflect hematoxylin and eosin-stained sections. Parts (C) and (D) reflect Congo red-stained sections. The gastric biopsy demonstrated pink amorphous material that showed apple-green birefringence on Congo red staining, which was consistent with amyloid deposition. For amyloid sub-typing, the specimen was sent for liquid chromatography tandem mass spectrometry, which showed a peptide profile consistent with AL (lambda)-type amyloid deposition. 


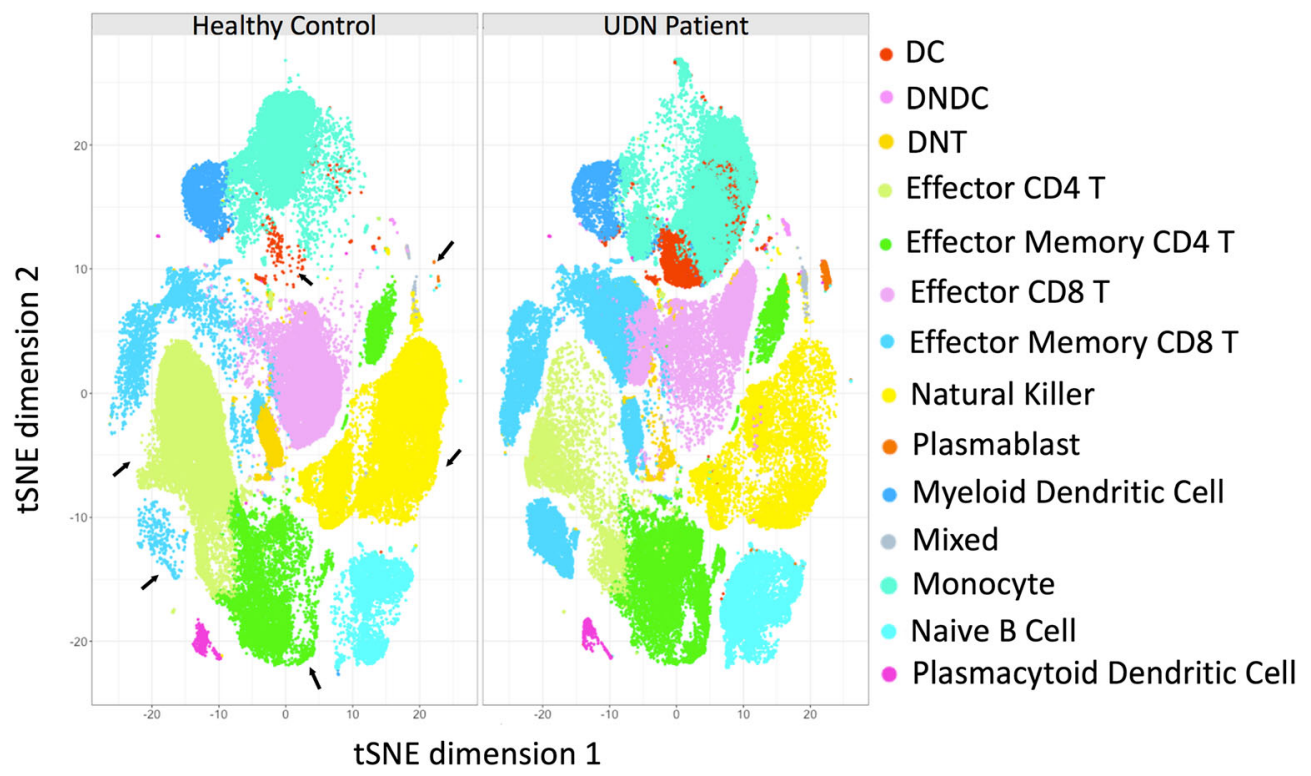

Figure 3 Change in immune cell populations between samples from a healthy control patient and our patient. CyTOF immunophenotyping of PBMCs from healthy control (A) and our patient (B). tSNE dimensionality reduced data from healthy and disease PBMCs are plotted, where each dot represents a cell. The numbers indicate the different clusters identified by the FlowSOM algorithm. The dots are color coded by the type of immune cell they represent. Clusters that varied the most between healthy and disease sample are indicated with arrows. NK natural killer, PB plasmablast, DC dendritic cell, mDC myeloid DC, pDC plasmacytoid DC, DN double negative, Eff. effector, Mem. memory.

further research on new treatments. Recent evidence suggests that botulinum toxin can be effective in treating inclusion body myositis-associated dysphagia. ${ }^{21}$

Indeed, in this era of increasingly personalized medicine, ${ }^{1,}$ ${ }^{22}$ research on new treatments will undoubtedly require more precise categorization of individual patient pathology and disease mechanisms. Given our patient's unique presentation and access to non-clinically available research tests through the UDN, we sought to more precisely define her disease process using advanced immunophenotyping. Recent research suggests that there is a distinct cell-based immunologic signature in Sjogren's patients; furthermore, immunologic data can be used to stratify subsets of patients (i.e., for disease severity). ${ }^{23}$ In Figures 3 and S4, we compared immune cell populations in our patient's blood with a control (non-Sjogren's syndrome) sample. In our patient, we provide confirmation that Sjogren's is indeed associated with increased plasmablasts, effector memory CD8+ T cells, and effector memory CD4+ T cells and decreased plasmacytoid dendritic cells.

Detailed information on research methods and additional figures are included in the online-only appendix (Appendix A). When applied to large samples of patients, these methods may lead to precision diagnosis, more individualized therapies, and more precise prognostic information. ${ }^{24}$ While the specific tests in Appendix A are not currently clinically available, we believe that they provide an important glimpse into immunophenotyping that may eventually be integrated into clinical practice. The highlighted tests represent a more granular version of clinically available tests. For example, a complete blood count (CBC) with differential is a common form of basic immunophenotyping, and other quantitative immunophenotyping tests such as immunoglobulin concentrations and CD4 counts are used for clinical treatment decisions.

Moreover, we hope to highlight some of the research-based testing available via the UDN. In this particular case, while the UDN referral was clearly productive, it may not have been inevitable. However, in other cases, referrals to the UDN may be essential in order to access necessary non-clinically available testing such as whole genome sequencing, RNA sequencing, or the immunophenotyping that occurred in our case. We hope to encourage further referrals and collaboration between primary care providers and the UDN.

\section{CONCLUSION}

We report a novel case involving the trio of Sjogren's syndrome, IBM, and AL amyloidosis. Sjogren's syndrome is one of the most common rheumatologic disorders. While IBM is relatively rare, it is the most common inflammatory myopathy in adults over 50 . Therefore, familiarity with these disorders can help the primary care clinician facilitate and expedite appropriate testing, diagnosis, sub-specialist referral, and treatment.

\section{Acknowledgements:}

Contributors: We would like to gratefully acknowledge Dr. Holden Maecker and Dr. Rosemary Fernandez for their work on the immunophenotyping for our patient. Members of the Undiagnosed Diseases Network are listed in the appendix. 
Corresponding Author: Jason Hom, MD; Division of Hospital Medicine, Department of Medicine Stanford University School of Medicine, Stanford, CA, USA (e-mail:jasonhom@stanford.edu).

Funders This work was funded by the NIH Common Fund grant U01HG007708.

\section{Compliance with Ethical Standards:}

Conflict of Interest: The authors declare that they do not have a conflict of interest.

IRB Approval: Our study was approved by Stanford's IRB.

\section{REFERENCES}

1. Gahl WA, Wise AL, Ashley EA. The Undiagnosed Diseases Network of the National Institutes of Health: A National Extension. JAMA, 2015;314(17):1797-8

2. Kanellopoulos P, Baltoyiannis C, Tzioufas AG. Primary Sjogren's syndrome associated with inclusion body myositis. Rheumatology (Oxford), 2002;41(4):440-4

3. Rojana-udomsart A, et al.The association of sporadic inclusion body myositis and Sjogren's syndrome in carriers of HLA-DR3 and the 8.1 MHC ancestral haplotype. Clin Neurol Neurosurg. 2011;113(7):559-63

4. Perlat A, et al. Systemic light chain amyloidosis and Sjogren syndrome: an uncommon association. In: Amyloid. England; 2009:181-2

5. Dalakas MC, et al. Inclusion body myositis and paraproteinemia: incidence and immunopathologic correlations. Ann Neurol. 1997;41(1):100-4

6. Dimachkie MM, Barohn RJ. Inclusion Body Myositis. Neurol Clin. 2014;32(3):629-46

7. Cruz-Tapias $\mathbf{P}$, et al. HLA and Sjogren's syndrome susceptibility. A metaanalysis of worldwide studies. Autoimmun Rev. 2012;11(4):281-7

8. Badrising UA, et al. Associations with autoimmune disorders and HLA class I and II antigens in inclusion body myositis. Neurology. 2004;63(12):2396-8

9. Misterska-Skora $\mathbf{M}$, et al. Inclusion body myositis associated with Sjogren's syndrome. Rheumatol Int. 2013;33(12):3083-6
10. Benveniste $\mathbf{O}$, et al. Amyloid deposits and inflammatory infiltrates in sporadic inclusion body myositis: the inflammatory egg comes before the degenerative chicken. Acta Neuropathol. 2015;129(5): p. 611-24

11. Parker KC. et al. Fast-twitch sarcomeric and glycolytic enzyme protein loss in inclusion body myositis. Muscle Nerve. 2009;39(6):739-53

12. Lloyd TE, et al. Evaluation and construction of diagnostic criteria for inclusion body myositis. Neurology. 2014;83(5):426-33

13. Cox FM, et al. A 12-year follow-up in sporadic inclusion body myositis: an end stage with major disabilities. Brain. 2011;134(Pt 11):3167-75

14. Benveniste $\mathbf{O}$, et al. Long-term observational study of sporadic inclusion body myositis. Brain. 2011;134(Pt 11):3176-84

15. Phillips BA, Zilko PJ, Mastaglia FL. Prevalence of sporadic inclusion body myositis in Western Australia. Muscle Nerve. 2000;23(6):970-2

16. Voermans NC, et al. Primary respiratory failure in inclusion body myositis. Neurology. 2004;63(11):2191-2

17. Graber ML, Mathew A. Performance of a web-based clinical diagnosis support system for internists. J Gen Intern Med. 2008;23 Suppl 1:37-40

18. Bond WF, et al. Differential diagnosis generators: an evaluation of currently available computer programs. J Gen Intern Med. 2012;27(2):213-9

19. Riches N, et al. The Effectiveness of Electronic Differential Diagnoses (DDX) Generators: A Systematic Review and Meta-Analysis. PLoS One. 2016;11(3):e0148991

20. Derk CT, et al. Inclusion body myositis in connective tissue disorders: case report and review of the literature. Clin Rheumatol. 2003;22(45):324-8

21. Schrey A, et al. Botulinum toxin alleviates dysphagia of patients with inclusion body myositis. J Neurol Sci. 2017;380:142-147

22. Ramoni RB, et al. The Undiagnosed Diseases Network: Accelerating Discovery about Health and Disease. Am J Hum Genet. 2017;100(2):185192

23. Mingueneau M, et al. Cytometry by time-of-flight immunophenotyping identifies a blood Sjogren's signature correlating with disease activity and glandular inflammation. J Allergy Clin Immunol. 2016;137(6):18091821.e12

24. Renaudineau Y. Immunophenotyping As a New Tool for Classification and Monitoring of Systemic Autoimmune Diseases. Clin Rev Allergy Immunol. 2017;53(2):177-180

Publisher's Note: Springer Nature remains neutral with regard to jurisdictional claims in published maps and institutional affiliations. 